\title{
Sciendo
}

DOI: $10.1515 /$ sspjce-2019-0019

\section{Application of the theory of limit analysis for the study of the behavior of the ground anchor in homogeneous soil}

\author{
Feriel Mekki', Mohamed Meksaouine ${ }^{2}$ \\ ${ }^{1}$ Department of civil engineering and hydraulics, University of 08 May 1945, P.B.No:401, Guelma, 24000, Algeria \\ ${ }^{2}$ Department of hydraulics, University of Badji Mokhtar, P.B. №12, Sidi Amar, Annaba, 23000, Algeria \\ e-mail: feriel.mekki@univ-annaba.dz,mohamed.meksaouine@univ-annaba.dz
}

\begin{abstract}
Ground anchors are devices used in many types of structures (on shore and offshore constructions); they can be oriented at different inclinations. Their roles are to resist tensile forces, stabilize and restrain the movement of engineered structure. The objective of this study is to propose a model which represents the mechanism of rupture of homogeneous soil (granular medium) around a single inclined ground anchor when it is tensioned and the determination of the collapse load thanks of the theory of limit analysis based on the kinematic approach in the case of criterion of Mohr-Coulomb taking into account of the mobilization of the soil in abutment in reaction to the loading.
\end{abstract}

Key words: collapse load, single ground anchor, kinematic approach, limit analysis, mechanism of rupture, Mohr-Coulomb criterion.

\section{Introduction}

Ground anchors also known as earth anchors or mechanical anchors have been used in the construction since the turn of the $19^{\text {th }}$ century. The first application dates from 1934 in Cheurfa dam in Algeria. Their roles are to mobilize and transfer the required resisting force from the structural element to the soil. The main concern in the design and the dimensioning of the ground anchors is the determination of the capacity which they can support to prevent the rupture of the anchored structures and to ensure its stability with a high security level. The determination of the collapse load depends on several factors such as: the orientation of the ground anchors and their characteristics (the free length, the bonded length and its thickness), the type of soil (cohesionless,cohesive) and its characteristics: (angle of friction, cohesion and dilatancy), resistance of the steel, dimensions of the perforations, characteristics and quantity of the grout, irregularity of the shape of the bulb.Very few theoretical studies have been performed to determine the collapse load of ground anchors in sandy soil; empirical relationships were suggested to correlate the failure load of a single ground anchor with its 
geometric properties and with the characteristics of the surrounding soil [1], [2], [3], [4], [5],[6],[7],[8]. In addition, analytical models were developed to predict the ultimate load of ground anchors [9], [10] [11], [12], [13]; but this researches did not include the effect of inclination of the ground anchor and the angle of the slope of the soil. Through this study, we propose to make a contribution to the determination of the mechanism of failure of a single inclined ground anchor at the time of tension in granular medium and to examine the effect of its inclination; the friction angle of the soil and the angle of the slope on the limit load with the use of a theoretical approach which is the theory of limit analysis using its kinematic approach.

\section{The theory of limit analysis}

For the study of the behavior of a single inclined ground anchor in homogeneous isotropic soil (cohesionless soil) we used a theoretical approach, which is the limit analysis in the case of the Mohr-Coulomb criterion. The theory of limit analysis is a simple method used to predict the capacity of structures to support a determined load. It relies on knowledge of the geometry of a given system, of the loading mode which is applied, resistance capacities of the constituent materials. The theory of limit analysis is based on two complementary approaches: "the static approach" and "the kinematic approach». The static approach: it stipulates that the collapse load calculated from a statically admissible stress field is a lower limit (lower bound) of the real limit load. The kinematic approach: it stipulates that the collapse load calculated from a kinematically admissible stress field is an upper limit (upper bound) of the real limit load.

\section{Development of the model by the kinematic approach of limit analysis and assumptions of calculation}

A single, inclined ground anchor of length (L) is placed in homogenous isotropic noncohesive soil having a friction angle $(\varphi)$ and unit weight $(\gamma)$. The ground anchor is inclined at an angle $(\eta)$ and the sloping surface is inclined at an angle $(\theta)$ with the horizontal. The ground anchor is subjected to a static load (F) with its point of application coinciding with the axis of the ground anchor. The anchor-soil roughness angle is higher than the friction angle $(\delta \geq \varphi)$ [14].The soil mass was assumed to obey Mohr-Coulomb's failure criterion and an associated flow rule taking into account of the mobilization of the ground in abutment in reaction to the loading. The objective is to determine by the upper bound of limit analysis the failure mechanism and the collapse load during the tensioning of the ground anchor. 


\section{Mechanism of failure of the ground surrounding the inclined ground anchor in the plane according to Mohr- Coulomb criterion}

The collapse mechanism presented in figure 1 is composed of triangular rigid block (DAB ) described by the parameter $(\beta)$ limited by the straight lines of rupture $(\mathrm{AD}),(\mathrm{AB})$ and the tilted free surface .The block $(\mathrm{DAB})$ move with velocity $\left(\mathrm{V}_{1}\right)$ making an angle $(\varphi)$ with the rupture lines $(A D)$ and $(A B)$,the ground move with the velocity $\left(V_{0}\right)$. The relative velocity $\left(V_{01}\right)$ is also inclined at an angle $(\varphi)$ with the ground anchor $(A O)$. All the velocities can be computed in terms of $\left(\mathrm{V}_{0}\right)$ following the velocity hodograph, as shown in in figure 2.

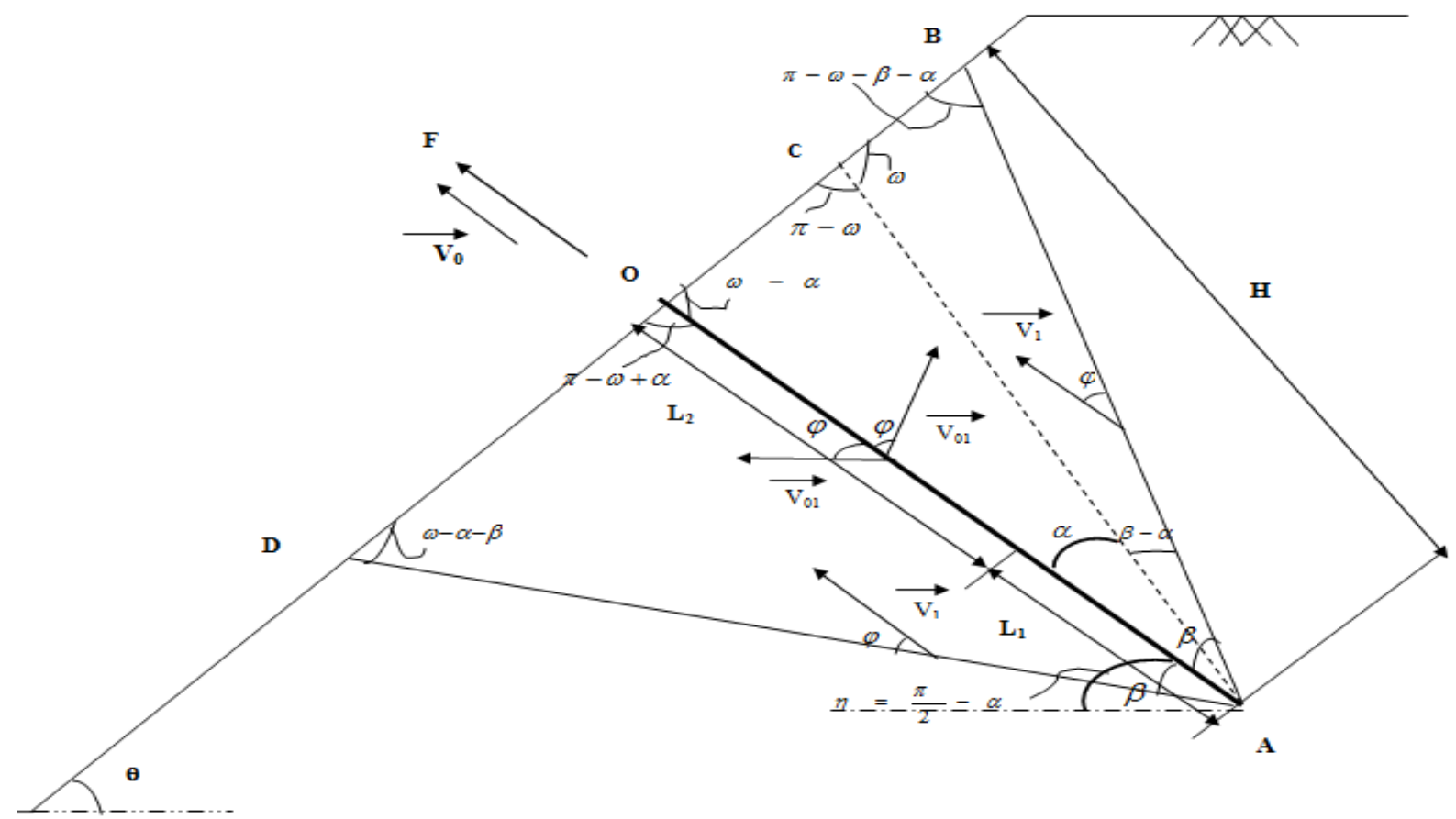

Figure 1: Mechanism of rupture of the ground surrounding the tilted ground anchor $(\delta \geq \varphi)$ in plan according to Mohr-Coulomb criterion

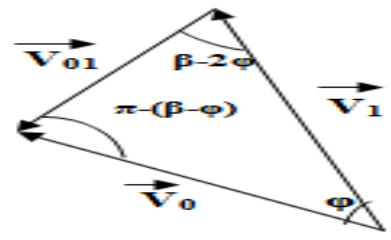

Figure 2: Velocity hodograph for failure mechanism

The velocity of the block:

$$
V_{1}=\frac{V_{0} \cdot \sin (\beta-\varphi)}{\sin (\beta-2 \varphi)}
$$

The relative velocity ground anchor-soil:

$$
V_{01}=\frac{V_{0} \cdot \sin (\varphi)}{\sin (\beta-2 \varphi)}
$$


The lines of rupture:

$$
L_{O A}=L_{1}+L_{2}=L
$$

L: the total length of the ground anchor

$\mathrm{L}_{1}$ : the bonded length

$\mathrm{L}_{2}$ : the unbonded length

$\theta:$ the angle of inclination of sloping surface

$\eta$ : the angle of inclination of the ground anchor

$$
\begin{gathered}
\omega=\frac{\pi}{2}+\theta \\
\alpha=\frac{\pi}{2}-\eta \\
L_{O C}=\frac{L \cdot \sin (\alpha)}{\sin (\pi-\alpha)}
\end{gathered}
$$

The internal dissipation of energy respectively along the lines of rupture $\left(\mathrm{L}_{\mathrm{AB}}\right),\left(\mathrm{L}_{\mathrm{AD}}\right)$ and along the ground anchor of length (LOA) is equal to:

$$
\begin{gathered}
E_{A B}=c \cdot L_{A B} \cdot V_{1} \cdot \cos (\varphi) \Rightarrow \quad E_{A B}=\frac{c \cdot L \cdot \sin (\omega-\alpha) \cdot V_{0} \cdot \sin (\beta-\varphi) \cdot \cos (\varphi)}{\sin (\pi-\omega-\beta+\alpha) \cdot \sin (\beta-2 \varphi)} \\
E_{A D}=c \cdot L_{A D} \cdot V_{1} \cdot \cos (\varphi) \Rightarrow E_{A D}=\frac{c V_{0} \cdot L \cdot \sin (\pi-\omega+\alpha) \sin (\beta-\varphi) \cdot \cos (\varphi)}{\sin (\omega-\alpha-\beta) \cdot \sin (\beta-2 \varphi)} \\
E_{O A}=c \cdot L_{1} \cdot V_{01} \cdot \cos (\varphi)=>\quad E_{O A}=\frac{c \cdot L_{1} \cdot V_{0} \cdot \sin (\varphi) \cos (\varphi)}{\sin (\beta-2 \varphi)}
\end{gathered}
$$

For a rough ground anchor $(\delta \geq \varphi)$, the dissipation of energy by friction of a non-cohesive soil is equal to zero.

The weight of solid masses $(\mathrm{OAD})$ and $(\mathrm{OAB})$ is given by following expressions:

$$
\begin{gathered}
W_{O A D}=\frac{\gamma \cdot L^{2} \cdot \sin (\pi-\omega+\alpha) \cdot \sin (\beta)}{2 \cdot \sin (\omega-\alpha-\beta) \cdot \sin \left(\frac{\pi}{2}\right)} \\
W_{O A B}=\frac{\gamma \cdot L^{2} \cdot \sin (\omega-\alpha) \cdot \sin (\beta)}{2 \cdot \sin (\pi-\omega-\beta+\alpha) \cdot \sin \left(\frac{\pi}{2}\right)}
\end{gathered}
$$

c: the cohesion of the soil

$\gamma:$ the unit weight of the soil

The work of the external forces:

The work of the gravitational force:

$$
T=F \times V_{0}
$$

$$
\begin{gathered}
T_{O A B}=W_{O A B} \times V_{1} \times \cos (\pi-\beta+\alpha+\varphi) \\
T_{D A O}=W_{D A O} \times V_{1} \times \cos (\pi-\beta-\alpha-\varphi)
\end{gathered}
$$


Equating the internal rate of work done by external forces to internal dissipation of energy; the value of $(\mathrm{F})$ can be obtained:

$$
\begin{aligned}
& F=\frac{c \cdot L \cdot \sin (\omega-\alpha) \cdot \sin (\beta-\varphi) \cdot \cos (\varphi)}{\sin (\pi-\omega-\beta+\alpha) \cdot \sin (\beta-2 \varphi)}+\frac{c \cdot L_{1} \cdot \sin (\varphi) \cdot \cos (\varphi)}{\sin (\beta-2 \varphi)}+\frac{c \cdot L \cdot \sin (\pi-\omega+\alpha) \cdot \sin (\beta-\varphi) \cdot \cos (\varphi)}{\sin (\omega-\alpha-\beta) \cdot \sin (\beta-2 \varphi)}+ \\
& \frac{\gamma \cdot L^{2} \cdot \sin (\omega-\beta) \cdot \sin (\beta) \cdot \cos (\pi-\beta+\alpha-\varphi) \cdot \sin (\beta-\varphi)}{2 \cdot \sin (\pi-\omega-\beta+\alpha) \sin \left(\frac{\pi}{2}\right) \cdot \sin (\beta-2 \varphi)}+\frac{\gamma \cdot L^{2} \cdot \sin (\beta) \sin (\pi-\omega+\alpha) \cdot \cos (\pi-\beta-\alpha-\varphi) \cdot \sin (\beta-\varphi)}{2 \cdot \sin (\omega-\alpha-\beta) \cdot \sin \left(\frac{\pi}{2}\right) \sin (\beta-2 \varphi)}
\end{aligned}
$$

The analysis of equations and results is processed by MATHCAD software.

\section{The material used}

To estimate the collapse load of a single inclined ground anchor placed in granular medium; we have used Hostun sand. It is sand with tight granulometry whose characteristics appear in table 1 .

Table 1: Physical characteristic of the sand

\begin{tabular}{|l|c|c|c|c|}
\hline Designation & \multicolumn{2}{|c|}{$\begin{array}{c}\text { Dimensions in } \\
\mathrm{mm}\end{array}$} & \multicolumn{2}{c|}{ Void ratio } \\
\cline { 2 - 5 } & $\mathrm{d}_{\min }$ & $\mathrm{d}_{\max }$ & $\mathrm{e}_{\min }$ & $\mathrm{e}_{\max }$ \\
\hline Sand & 0.3 & 1 & 0.607 & 0.885 \\
\hline
\end{tabular}

The relationship between the density of the sand and its friction angle is put in form of polynomial of the second degree [15].

$$
\varphi=-97.0424 \times D^{2}+368.8507 \times D-304.919
$$

The soil parameters used in this study: the unit weight $\left(\gamma=1570 \mathrm{~kg} / \mathrm{m}^{3}\right)$, the friction angle $\left(\varphi=35^{\circ}\right)$.

\subsection{Application with software Mathcad}

The model is introduced into the software MATHCAD which will allow us the visualization of the curve of the collapse load $(\mathrm{F})$ as shown in figure 3.

Data:

$\mathrm{L}=12 \mathrm{~m}, \mathrm{~L}_{1}=4 \mathrm{~m}, \mathrm{c}=0 \mathrm{~kg} / \mathrm{m}^{2}, \gamma=1570 \mathrm{~kg} / \mathrm{m}^{3}, \varphi=35^{\circ}, \mathrm{V}_{0}=1 \mathrm{~m} / \mathrm{s}, \theta=25^{\circ}, \eta=20^{\circ}$

Boundary condition:

$\beta=1.7$

Given

$1.5<\beta<2$

Minimization:

$\operatorname{Minimize}(F, \beta)=1.828$

$\mathrm{F}(1.828)=2.437 \times 10^{5} \mathrm{~kg}$ 


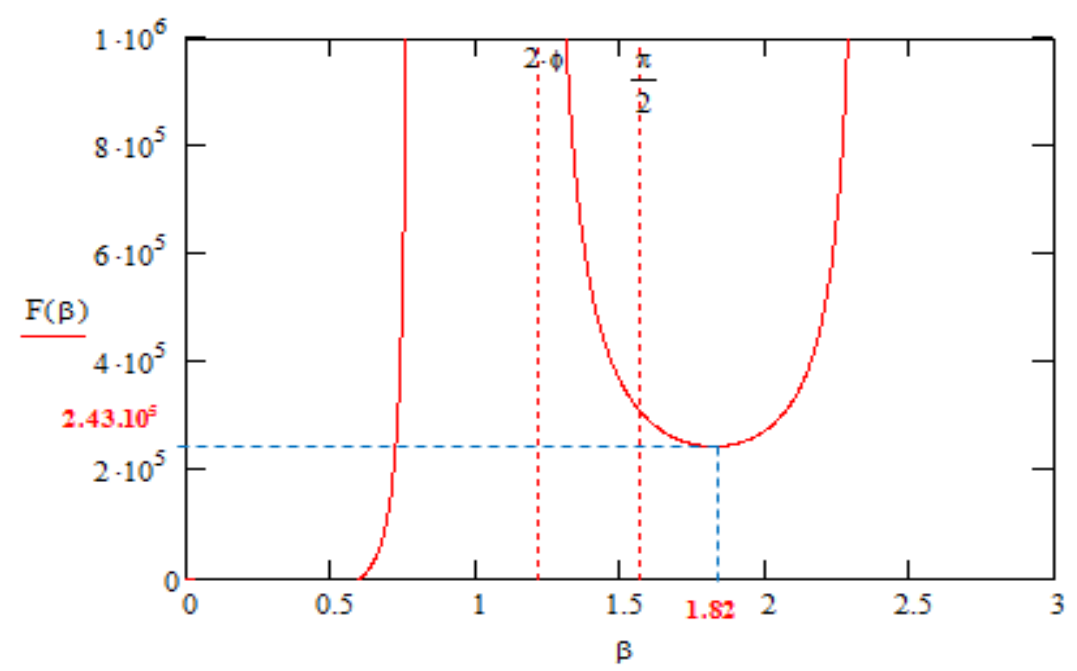

Figure 3: Visualization of the curve $\mathrm{F}(\beta)$ in the plan

The figure 3 represents the maximum effort of the model in the plan according to the parameter $(\beta)$ which has a concave form indicating a minimum.

\section{Mechanism of failure of the ground surrounding the inclined ground anchor in the space according to Mohr Coulomb criterion}

The mechanism of failure in the space is shown in figure 4 .At collapse; it is assumed that the ground anchor and the rigid block (DAB) move as a single rigid unit in the inclined direction with a velocity $\left(\mathrm{V}_{0}\right)$.

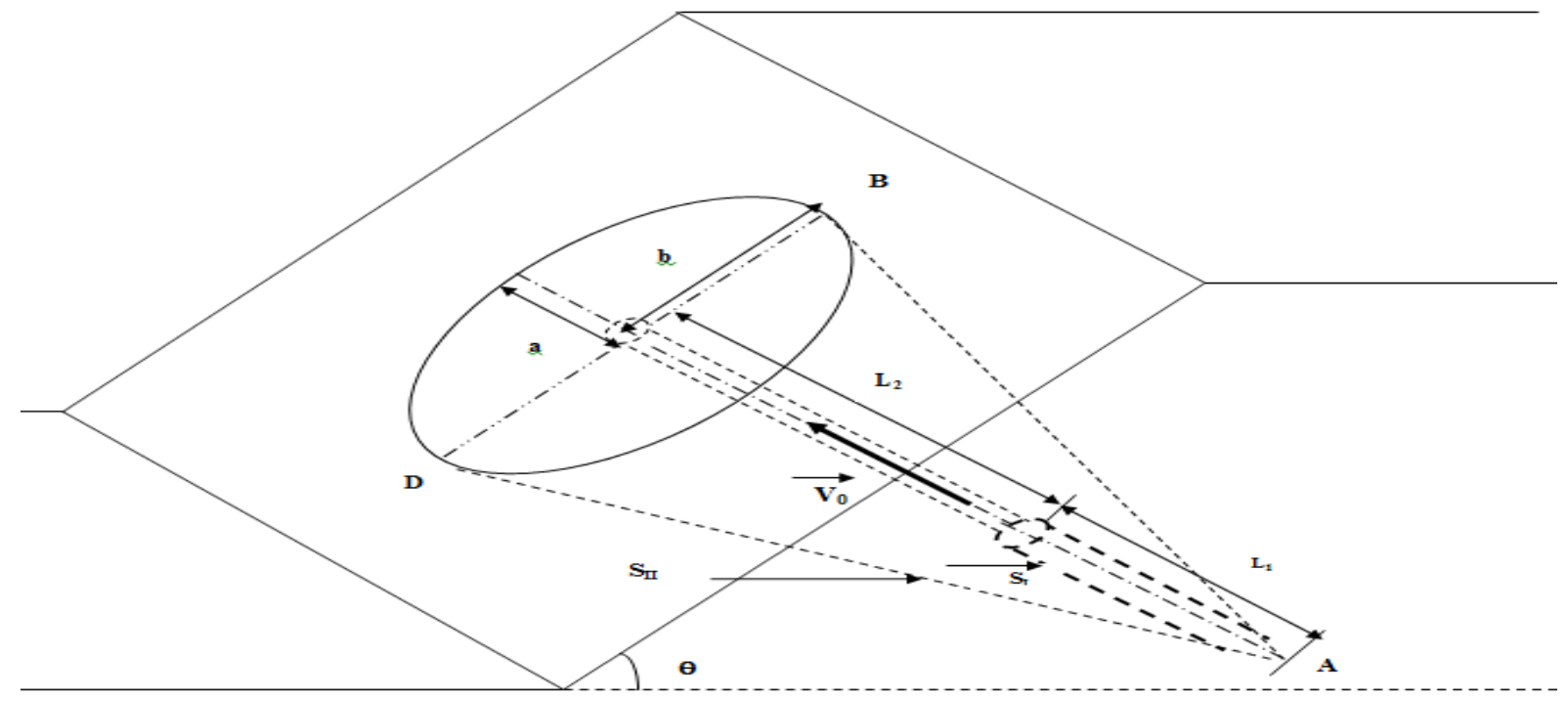

Figure 4: Mechanism of rupture of the ground surrounding the tilted anchor $(\delta \geq \varphi)$ in space according to Mohr-Coulomb criterion 
The velocities $\left(\mathrm{V}_{01}\right)$ and $\left(\mathrm{V}_{1}\right)$ are expressed respectively by the expressions (1) and (2).

The rupture surfaces:

$S_{I}:$ Side surface of the cylinder

$\mathrm{d}$ : the diameter of cylinder

$$
S_{I}=\pi \cdot d \cdot L_{1}
$$

$S_{I I}$ : Side surface of the cone

$$
\begin{gathered}
S_{I I}=\pi \cdot R \cdot H \\
H=L \sin (\omega-\alpha) \\
L_{D B}=\frac{H \cdot \sin (2 \beta)}{\cos (\alpha+\beta) \cdot \cos (\alpha-\beta)} \\
R=\frac{L_{D B}}{2}=b=\frac{H \cdot \sin (2 \beta)}{2 \cdot \cos (\alpha+\beta) \cdot \cos (\beta-\alpha)} \\
S_{I I}=\frac{\pi \cdot H^{2} \cdot \sin (2 \beta)}{2 \cdot \cos (\beta-\alpha) \cdot \cos (\alpha+\beta)}
\end{gathered}
$$

The internal dissipation of energy respectively along the rupture surfaces $\left(\mathrm{S}_{1}\right),\left(\mathrm{S}_{\mathrm{II}}\right)$ is equal to:

$$
\begin{gathered}
E_{O A}=c \cdot S_{I} \cdot V_{01} \cdot \cos (\varphi)=>E_{O A}=\frac{c \cdot \pi \cdot d \cdot L_{1} \cdot V_{0} \cdot \sin (\varphi) \cdot \cos (\varphi)}{\sin (\beta-2 \varphi)} \\
E_{D A}=c \cdot S_{I I} \cdot V_{1} \cdot \cos (\varphi)=>E_{D A}=\frac{c \cdot \pi \cdot H^{2} \cdot V_{0} \cdot \sin (2 \beta) \cdot \sin (\beta-\varphi) \cos (\varphi) \cdot}{2 \cdot \sin (\beta-2 \varphi) \cdot \cos (\beta-\alpha) \cdot \cos (\alpha+\beta) \cdot \sin (\beta-2 \varphi)}
\end{gathered}
$$

For a rough ground anchor $(\delta \geq \varphi)$, the dissipation of energy by friction of a non-cohesive soil is equal to zero.

The weight of solid masse (DAB):

$$
W_{D A B}=\frac{\pi \cdot \gamma \cdot a \cdot b \cdot H}{3}
$$

The surface of the ellipse:

The work of the external forces:

$$
\begin{gathered}
B=\pi \cdot a \cdot b \\
a=L . \operatorname{tg}(\beta) \\
b=\frac{H}{2}[\operatorname{tg}(\beta-\alpha)+\operatorname{tg}(\alpha+\beta)]
\end{gathered}
$$

The work of the gravitational force:

$$
T=F \times V_{0}
$$

$$
T_{D A B}=W_{D A B} \times V_{1} \times \cos (\pi-\alpha)
$$

Equating the internal rate of work done by external forces to internal dissipation of energy; the value of $(\mathrm{F})$ can be obtained:

$$
\begin{aligned}
& F=\frac{c \cdot \pi \cdot d \cdot L_{1} \cdot \sin (\varphi) \cdot \cos (\varphi)}{\sin (\beta-2 \varphi)}+\frac{c \cdot \pi \cdot H^{2} \cdot \sin (2 \beta) \cdot \sin (\beta-\varphi) \cdot \cos (\varphi)}{2 \cdot \sin (\beta-2 \varphi) \cdot \cos (\beta-\alpha) \cdot \cos (\alpha+\beta) \cdot \sin (\beta-2 \varphi)}+ \\
& \frac{\pi \cdot \gamma \cdot a \cdot b \cdot H \cdot \sin (\beta-\varphi) \cdot \cos (\pi-\alpha)}{3 \cdot \sin (\beta-2 \varphi)}
\end{aligned}
$$


The collapse load (F) is expressed in terms of non-dimensional uplift factor $\left(\mathrm{N}_{\gamma}\right)$ as defined herein:

$$
\begin{gathered}
F=N_{\gamma} \cdot \gamma \cdot L^{3} \\
N_{\gamma}=\frac{F}{\gamma \cdot L^{3}}
\end{gathered}
$$

\subsection{Application with software Mathcad}

The model is introduced into the software MATHCAD which will allow us the visualization of the curve of the collapse load (F) as shown in figure 5 .

Data:

$\mathrm{L}=12 \mathrm{~m}, \mathrm{~L}_{1}=4 \mathrm{~m}, \mathrm{~d}=0.12 \mathrm{~m}, \mathrm{c}=0 \mathrm{~kg} / \mathrm{m}^{2}, \gamma=1570 \mathrm{~kg} / \mathrm{m}^{3}, \varphi=35^{\circ}, \mathrm{V}_{0}=1 \mathrm{~m} / \mathrm{s}, \theta=25^{\circ}, \eta=20^{\circ}$

Boundary condition:

$\beta=2.4$

Given

$2<\beta<2.5$

Minimization:

$\operatorname{Minimize}(\mathrm{F}, \beta)=2.367$

$\mathrm{F}(2.367)=6.89 \times 10^{5} \mathrm{~kg}$

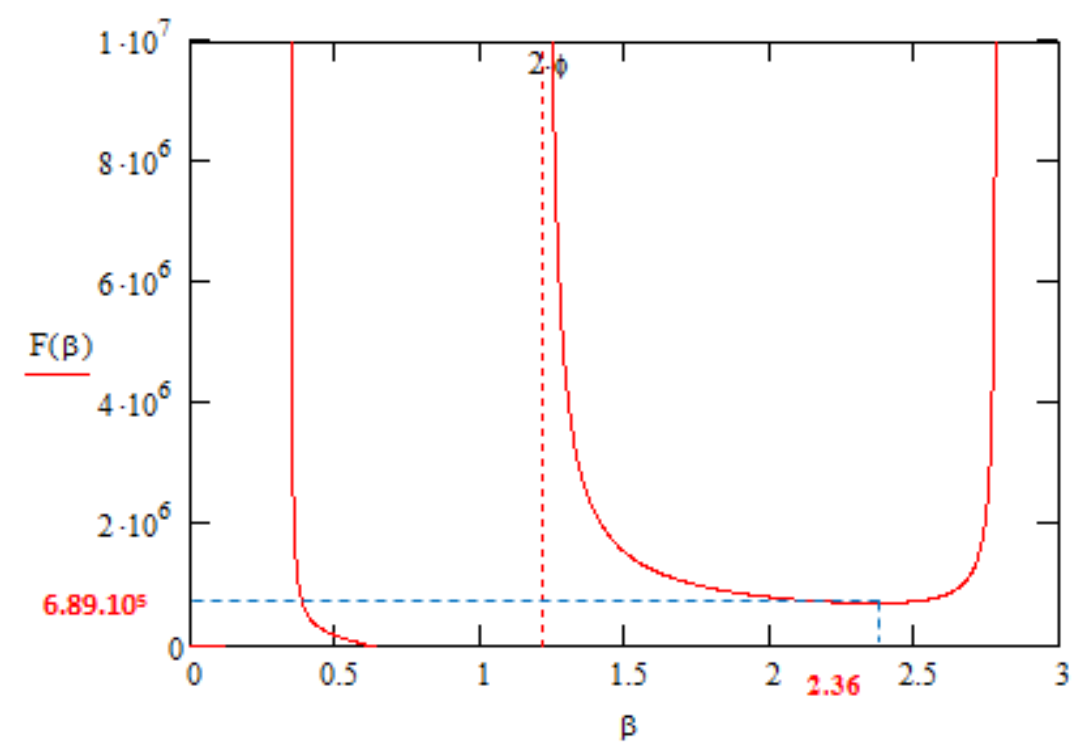

Figure 5: Visualization of the curve $F(\beta)$ in space

The figure 5 represents the maximum effort of the model in the space according to the parameter $(\beta)$ which has a concave form indicating a minimum. 


\section{Results and discussion}

We studied the effect of the friction angle $\left(\varphi\right.$ was varied from $30^{\circ}$ to $40^{\circ}$ with an interval of $1^{\circ}$ ), the effect of the angle of inclination of ground anchor ( $\eta$ was varied from $10^{\circ}$ to $30^{\circ}$ with an interval of $\left.5^{\circ}\right)$, and the effect of the angle of inclination of sloping surface ( $\theta$ was varied from $25^{\circ}$ to $33{ }^{\circ}$ with an interval of $\left.4^{\circ}\right)$ on the magnitude of the uplift factor $\left(\mathrm{N}_{\gamma}\right)$. The results obtained are shown in the figures below, from figure 6 to figure 13.

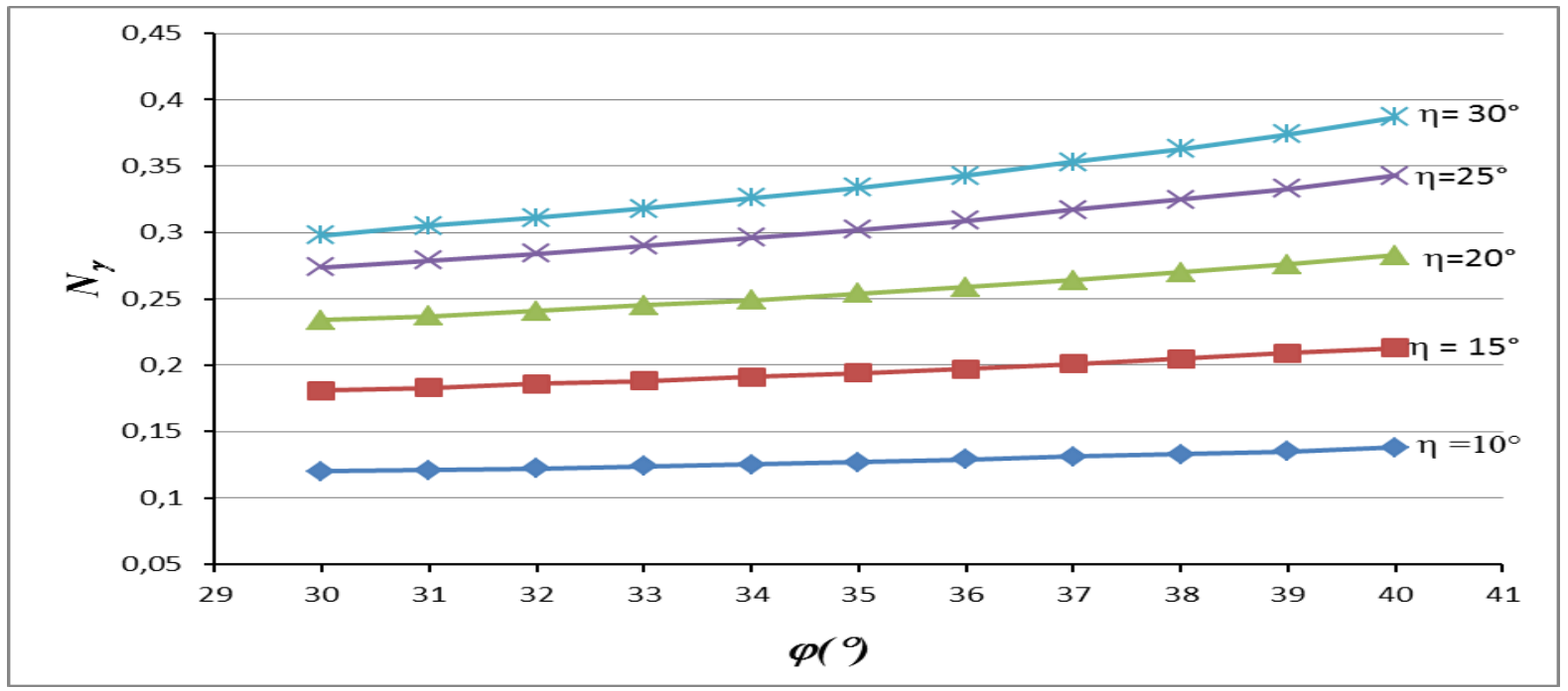

Figure 6: Evaluation of the uplift factor $\left(\mathrm{N}_{\gamma}\right)$ with $\eta$ and $\varphi$ for $\theta=25^{\circ}$

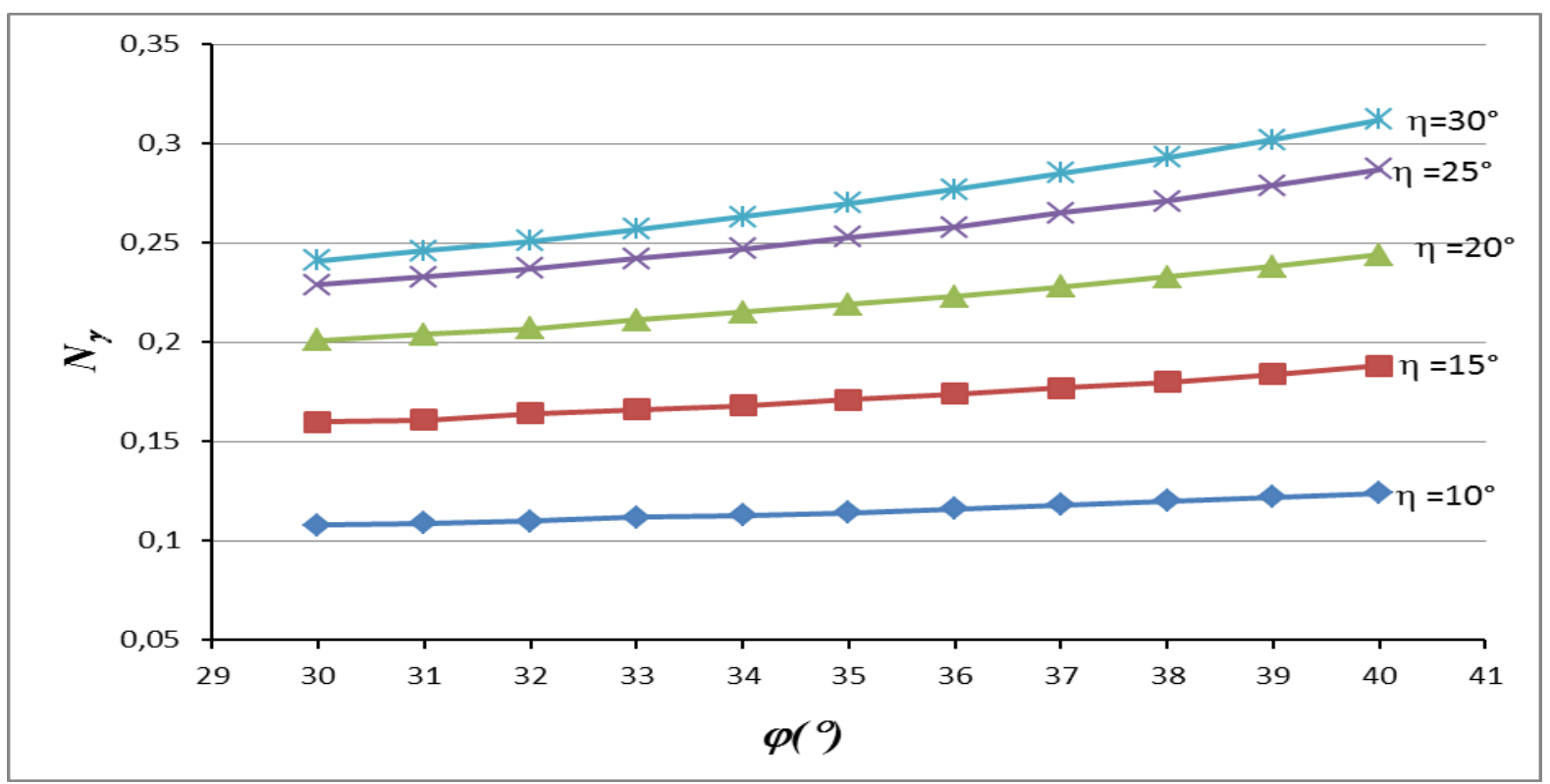

Figure 7: Evaluation of the uplift factor $\left(\mathrm{N}_{\gamma}\right)$ with $\eta$ and $\varphi$ for $\theta=29^{\circ}$ 


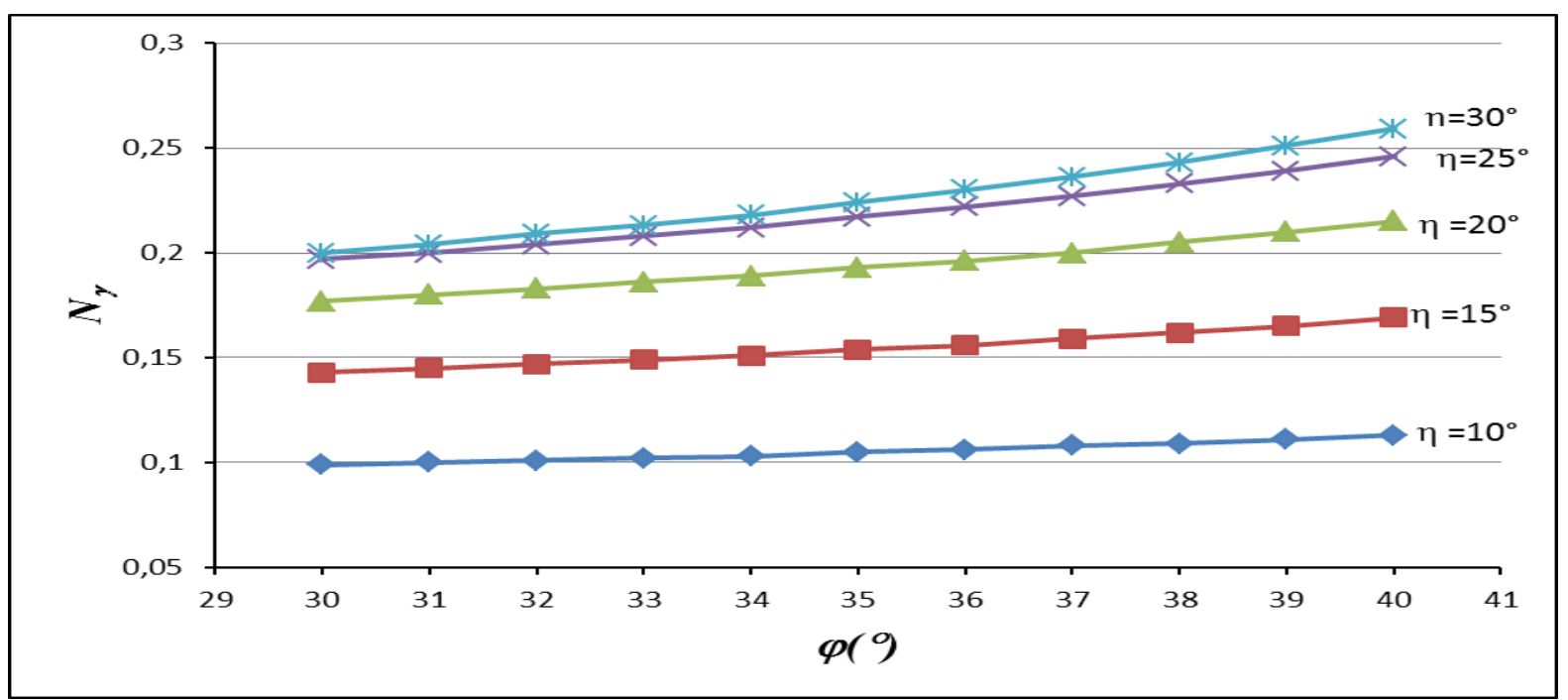

Figure 8: Evaluation of the uplift factor $\left(\mathrm{N}_{\gamma}\right)$ with $\eta$ and $\varphi$ for $\theta=33^{\circ}$

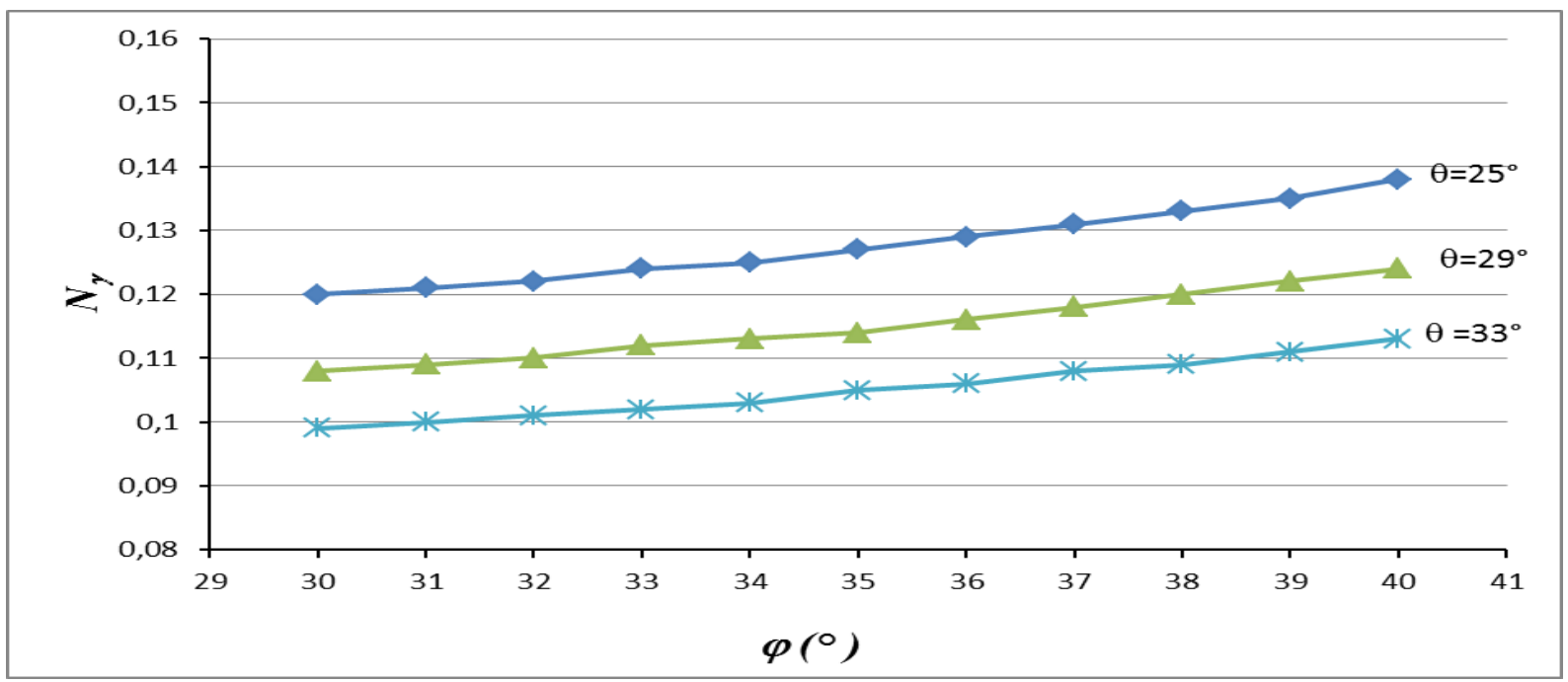

Figure 9: Evaluation of the uplift factor $\left(\mathrm{N}_{\gamma}\right)$ with $\theta$ and $\varphi$ for $\eta=10^{\circ}$ 


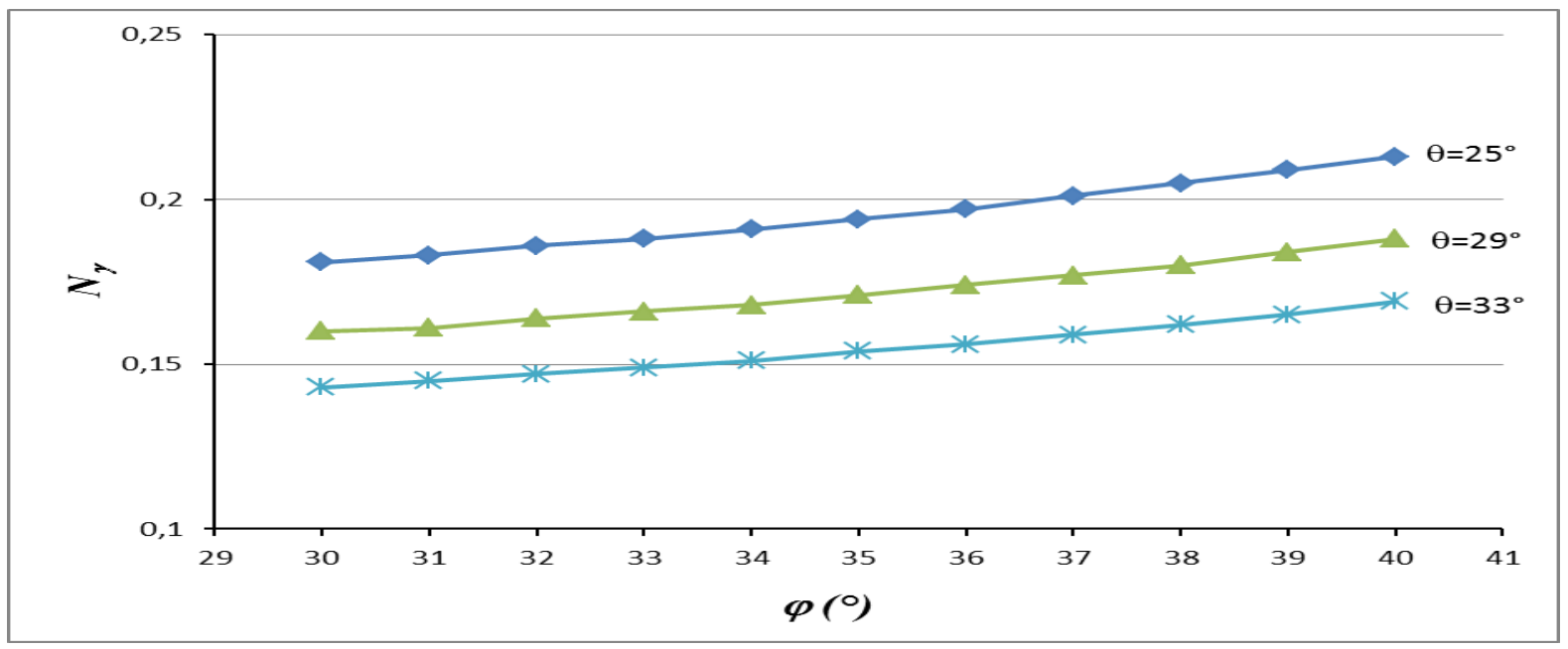

Figure 10: Evaluation of the uplift factor $\left(\mathrm{N}_{\gamma}\right)$ with $\theta$ and $\varphi$ for $\eta=15^{\circ}$

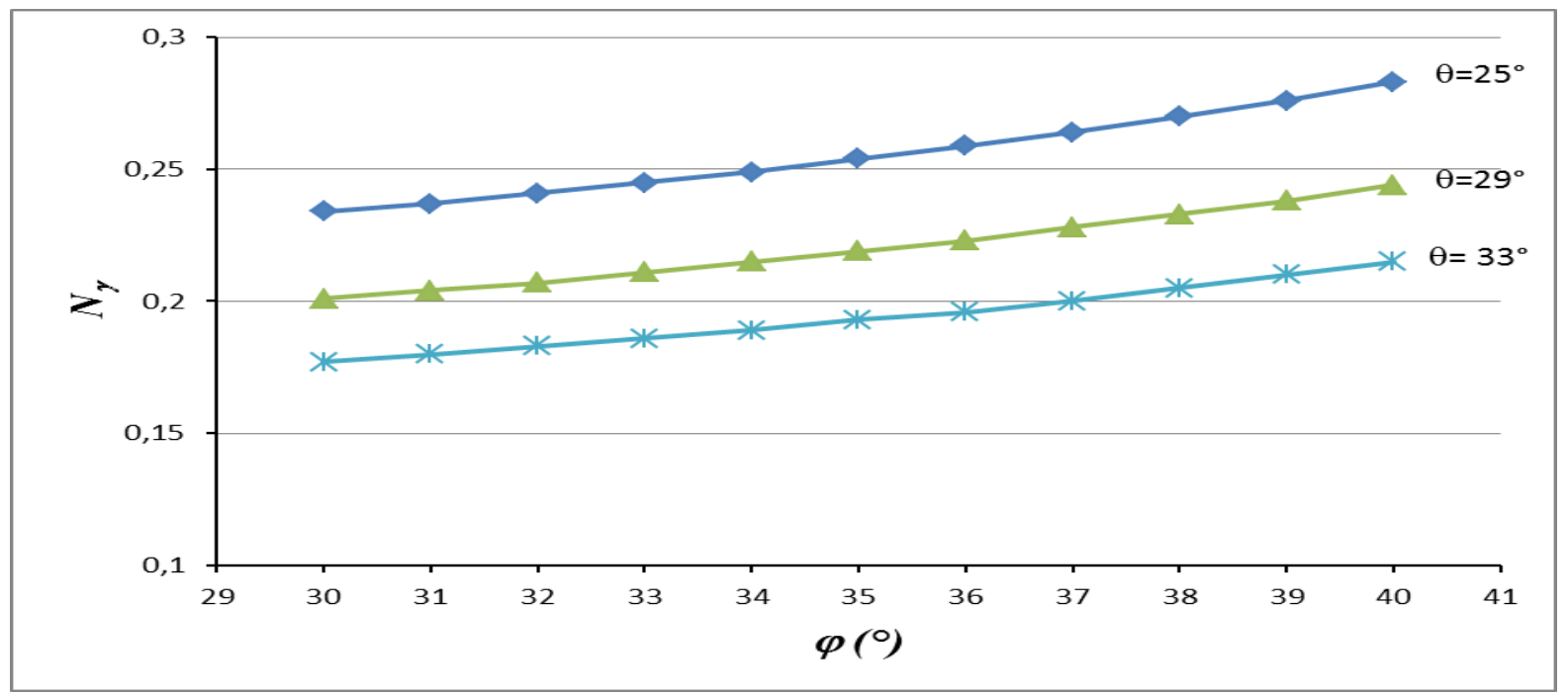

Figure 11: Evaluation of the uplift factor $\left(\mathrm{N}_{\gamma}\right)$ with $\theta$ and $\varphi$ for $\eta=20^{\circ}$ 


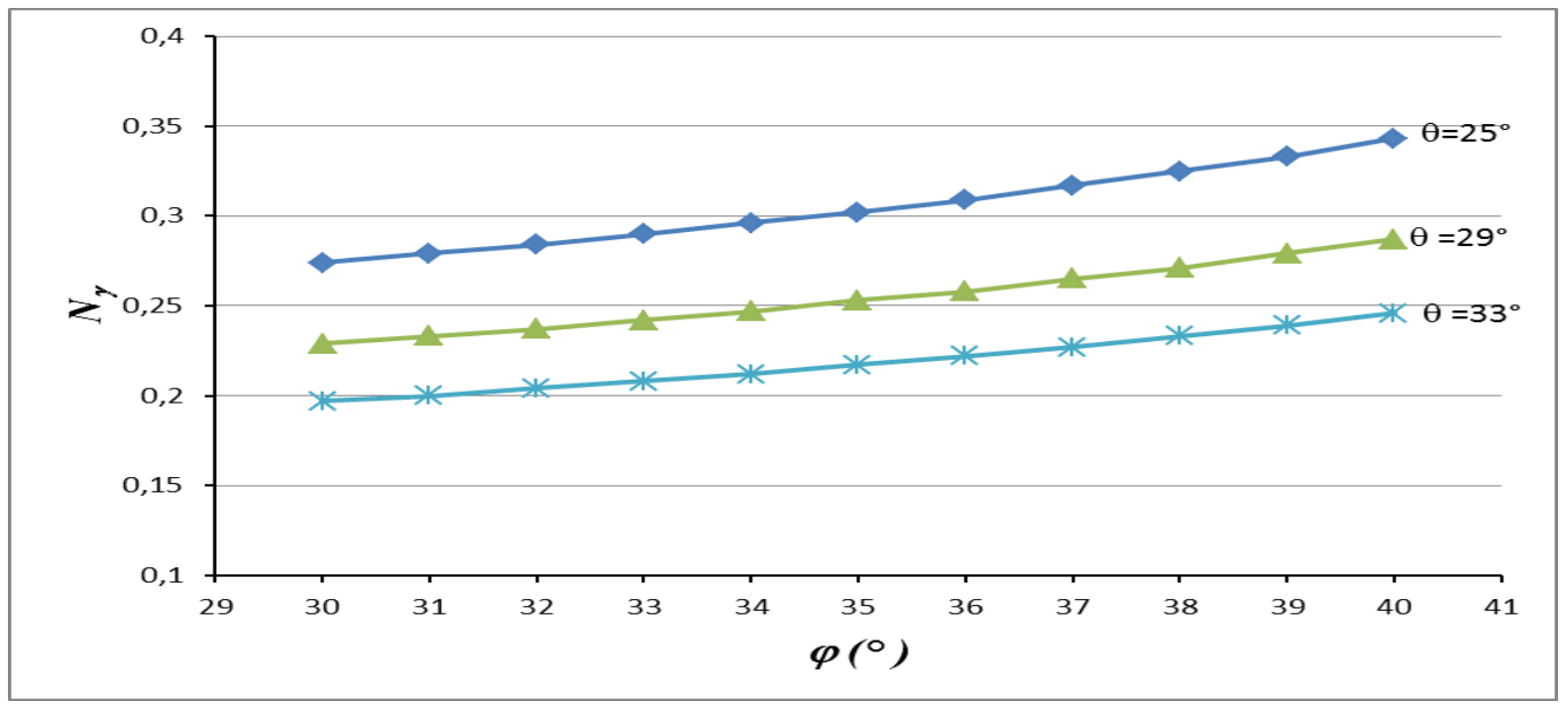

Figure 12: Evaluation of the uplift factor $\left(\mathrm{N}_{\gamma}\right)$ with $\theta$ and $\varphi$ for $\eta=25^{\circ}$

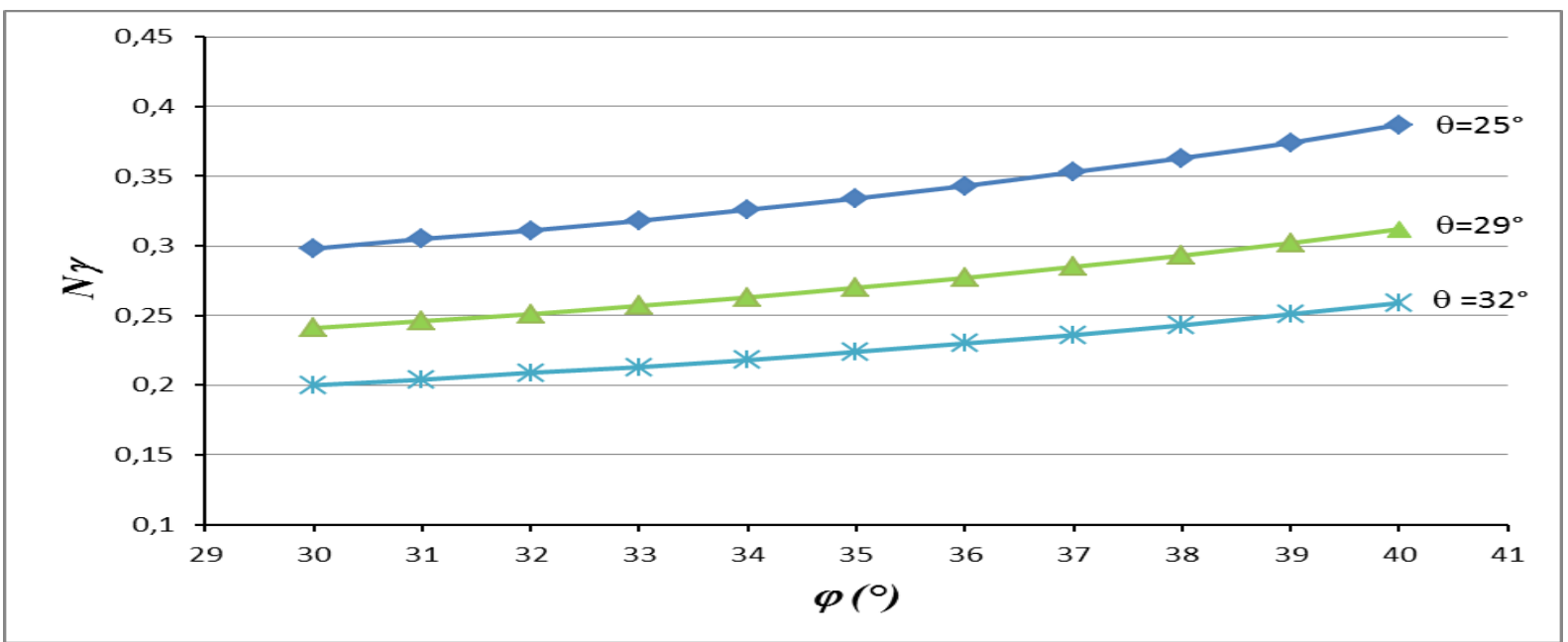

Figure 13: Evaluation of the uplift factor $\left(\mathrm{N}_{\gamma}\right)$ with $\theta$ and $\varphi$ for $\eta=30^{\circ}$

It can be observed from figures $6,7,8,9,10,11,12$ and 13 that the value of uplift factor $(\mathrm{N} \gamma)$ increases with the increase of the internal friction angle of the soil $(\varphi)$, with the inclination of the anchor $(\eta)$ and with the angle of the slope $(\theta)$. This implies that the collapse load increases with the evolution of these three parameters.

Figures 6 to 13 can be considered as abacuses for predicting the collapse load of an inclined ground anchor embedded in sloping ground composed of a cohesionless soil.

\section{Conclusion}

With the application of the kinematic approach of limit analysis, theoretical solutions have been generated for computing the ultimate load of a single inclined ground anchor buried in sloping ground (frictional soil) and subjected to static load for different soil friction angles 
with varying the ground anchor inclinations and the slope angles. The results obtained have been presented in terms of uplift factor $\left(\mathrm{N}_{\gamma}\right)$ in both graphical forms to facilitate their use in solving practical design problems.

Through this study the following results are founded:

- The shape of the curve $F(\beta)$ of the model in plane and space is concave indicating the existence of a minimum.

- The uplift factor $(\mathrm{N} \gamma)$ increases with the increase of the friction angle of the soil $(\varphi)$, the inclination of the ground anchor $(\eta)$ and the angle of the slope $(\theta)$.

- The elaborate model proposed in a single block undoubtedly overestimates the value of the limit load; but this is still a first limit value that the real value cannot exceed.

- The solutions developed in the present study are expected to be useful for purpose of design of inclined ground anchor embedded in sloping ground (sandy soil).

This work proposes a solution to this problem in the case of frictional soil never realized with this method that can be taken by other researchers.

\section{References}

[1] Hawkes, J. M. and Evans R. H. (1951). Bond stresses in reinforced concrete columns and beams. Structural Engineering, 29(12), 323-327.

[2] Phillips, S. H. E. (1970) Factors affecting design of anchorages in rock, Cementation Research Report R48/70.London, cementation research Ltd.

[3] Littlejohn, G. S. (1970). Soil anchors, Ground Engineering, Proceedings of an ICE Conference, ICE, London.

[4] Kramer. H. (1978). Determination of the carrying capacity of ground anchors with the correlation and regression analysis, Revue Française de Géotechnique, 3, 76-81.

[5] Hanna, T. (1982). Foundations in tension-ground anchors, Trans Tech Publications, Clausthal-Zellerfeld, Germany.

[6] Xanthakos, P.(1991). Ground anchors and anchored structures, John Wiley \& Sons, Inc,

[7] Barley, A.D. (1995). Theory and practice of the Single Bore Multiple Anchor System in Proceedings of the Anchors in Theory and Practice, International Symposium on Anchors in Theory and Practice, Saltzburg, Austria.

[8] Habib, P., Logcais, L., Clément, P., Dupeuble, P .(1995). Tirants d'ancrage (recommandations T.A.95), France, Eyrolles Editions.

[9] Mekki, F., Meksaouine, M. and Guenfoud, M. (2009). Theoretical study of the behaviour of anchors in homogeneous soil, International Review on modelling and simulations, 2, 221226.

[10] Mekki, F., Meksaouine, M. and Guenfoud, M. (2011). Etude théorique du comportement des tirants d'ancrages dans un sol pulvérulent, la revue Synthèse.,No. 23, 22-30.

[11] Rong, J. Z., Jun, J. Z., Pei, Y. L., Juan Z. and Shun Y. (2012). A method for predicting mechanical behavior of HPJG-Anchors part I: Mechanical characteristics and load transfer models, Computer and Geotechnics, 45, 62-73.

[12] Mekki, F. (2015).Etude théorique du comportement des tirants d'ancrages, Editions Universitaires Européennes. 
[13] Yang, Z., Chen, J., Zhang, H., Zhao, X., Li,H.(2018).Analytical calculation on the load displacement curve of grouts soil anchor, Journal of engineering science and technology review, 11 (3), 31-41.

[14] Chen, W.F. (1975). Limit Analysis and Soil Plasticity, Elsevier, Amesterdam, Netherland.

[15] Bourdeau, Y. (1977). Pushed non-cohesive soils. Influence characteristics of the grounds and model of test, $\mathrm{PhD}$ Thesis, I.N.S.A, Civil engineering. 